\title{
What R \&D Program for a fully non carbon energy supply by 2050?
}

Bernard Bigot

CEA, France

First of all, I would like to thank the organizers of this Conference for their kind invitation to participate and to give me the opportunity to present a possible $R \& D$ program for a long term sustainable supply of energy in Europe. I am deeply honored to attend this important event because I consider the energy supply as one of the major challenges our European societies will have to face in the near and medium term future. If we do not solve it properly, Europe will be largely handicapped in the economical and social world competition. I believe that relying on large, sustained and wide research and development activities shall help greatly to tackle the challenges. What are the challenges? The world has to deal with an expected increase of the energy needs, despite all the efforts we have to do for a better energy efficiency and for large energy savings and while all the existing energy technologies are in question: fossil, nuclear and renewables. There is no silver bullet. In fact, let's think about that if we offer the possibility of 2.5 tep/year/person for the 9 billions of inhabitants expected by 2050 in the world, that is to say half of the yearly energy consumption per capita to-day in Europe, we will have to increase the supply from $12 \mathrm{Gtep} /$ year to-day to nearly $23 \mathrm{Gtep} / \mathrm{year}$. This is a real doubling. Furthermore, if keeping the current energy mix, with $80 \%$ coming from fossil resources, we will strongly contribute to environmental and climate change and their damaging effects while these points are of increasing concern in the public opinion.

According to the French view, the constraints in terms of energy management are sustainability with a priority to free GHG emission energy sources, availability and security of supply, affordability and economical competitiveness, all of them lead to a reduction of fossil fuels. R\&D large and sustained efforts naturally ensue from the key issues to solve. 


\section{Let's consider more precisely the first question: Why and how does the EU energy mix need to change?}

To ensure its long term development, the world needs sustainable energy sources to make enough energy available for everybody at an acceptable price. In Europe today, energy supply relies mainly on fossil fuels, at nearly $80 \%$, as I said. Those fossil fuels are mainly imported and represent a heavy financial burden and a source of insecurity. Let's think about that France for the same amount of importations of gas and oil had to present $23 \mathrm{G} €$ in 2005 and $62 \mathrm{G} €$ in 2011. A tripling in 6 years! It is not sustainable at all. Massive use of fossil fuels is furthermore a threat for the environment, climate and health, and for our economies.

In EU, the main consumers of energy are housing and transport. This means that decarbonisation of EU energy mix will require acting primarily on electricity production and on energy efficiency in the transport and housing sectors, which means new energy production capacities, innovative energy management and energy storage.

In March 2007, the European Union stated the 3x20 objectives for 2020. More recently, in November 2011, the EC distributed a project on the "Energy Roadmap 2050", with more ambitious targets than those of the $3 \times 20$ objectives. The main outcome of the roadmap is that decarbonisation of the EU economy is possible with a cost rather higher than the present level of expenditures for energy (14\% against $9 \%$ of the EU GDP). All studied scenarios in the frame of the proposed roadmap include an increase of renewable energy sources, a decrease of fossil fuels with gas remaining a significant source and so requesting carbon capture and sequestration policy, a contribution of the nuclear in the range $2.5 \%$ to $21 \%$ depending on several hypothesis, and a strong improvement of energy efficiency. The renewable energy sources will require specific developments in terms of grids, storage capacities, production and connection infrastructures.

\section{I would like to defend some principles with regards to R\&D strategy:}

High uncertainties are attached to the proposed scenarios, particularly regarding energy sources and energy storage. In these conditions, it would be quite risky to bet on the development of a narrow range of energy sources. It is thus of first importance to be neutral with regards to the energy technologies provide they respect the global objectives we give us : sustainability, security of supply, economical competitiveness.... We have also to keep open a large spectrum of options. We have also to be open to structured partnership which may ease the collect of proper funding for R\&D. 
The development of ways to store energy massively would constitute a true revolution in our energy system organization. This is a long term objective. There is, according to me, no way to develop renewable energies if we do not find a way to store electrical energy and to take advantage of the complementarities of continuous, predictable energy supplies as nuclear and the intermittent ones as renewables. So, another principle I support is the development of synergies between the various carbon free energy sources. When we speak about renewable energy, we must favor local production and local storage including $\mathrm{H} 2$ storage and give priority to local consumption to avoid the grid bottlenecks and unreasonable over costs associated with energy transport.

\section{Research is key to implement those two principles:}

The needed technologies to achieve the transformation of our mix along these lines are often not mature. They will require a significant $R \& D$ effort including basic and technological research before being implemented. The $R \& D$ to be carried out will create the opportunity to develop collaboration and partnerships between European research organizations and industrials.

In France, "an alliance" called ANCRE which coordinates all energy research public actors has been created to reach the optimal energy mix, suited to our national needs by 2050 . At the European level, an alliance (EERA) has also been created to accelerate the development of innovative energy technologies to the point where they can be embedded in industry-driven research. To achieve this goal, EERA streamlines and coordinates national and European energy R\&D programs by conceiving and implementing Joint Programs of research in support of the SET-Plan priorities.

Funding all these researches at the appropriate level is a key issue since they require large amounts of money over long periods. I suggest that revenues from auctioning of $\mathrm{CO}_{2}$ allowances be used for this purpose. The amount of these revenues for a country like France is estimated of the order of $2 \mathrm{G} € /$ year. Such an investment in R\&D will fully change the energy landscape.

\section{Let us now focus on the main research fields to support:}

First, let me stress the need of complementarities between basic and technological research to overcome the numerous technological barriers and associated scientific challenges.

Basic research shall lead to the definition of concepts which future energy systems could rely on. 
Three specific fields are worth being highlighted: advanced materials in support to all energy developments, focused biology in support to the development of bio-energies, chemistry and industrial processes in valuing of carbon dioxide.

The development of advanced materials certainly represents one of the main challenges which could change the world of energy. The knowledge, at the molecular and atomic level of innovative materials structure and behavior is essential for the development of advanced materials. Such research will rely on large research infrastructures. For example, in the field of nuclear energy, the main issue stays in the material aging behavior under irradiation, high pressure and energy flux, rapid change in temperature. For the materials in future fast breeder reactors and mostly in fusion reactors, the irradiation and use constraint will be much higher, 10 times more than presently. This knowledge is crucial for safety and lifetime management of nuclear power plants.

In the field of renewable energies, the development of advanced materials has a similar key function on the system efficiency. In the solar field, complementarities between basic and technological research allowed to reach recently efficiency greater than $24 \%$ for photovoltaic cells at an industrial scale, which is a world class result.

For both nuclear and renewable energies, a particular attention should be devoted to spare and recycle strategic rare raw materials or replace them by abundant, easy to recycle and non toxic ones.

The second example of basic research I have mentioned, concerns the biology in support to the development of bio-technologies. The study of basic mechanisms of photosynthesis and metabolism of microalgae opens new opportunities for the production of third generation biofuel. Producing hydrogen by photocatalytic bio-inspired method requires the elaboration of systems able to capture photons and transform them into electron flux which then electrons oxidize water and reduce the resulting protons in hydrogen. In 2011, new promising results have been obtained by the CEA, on this approach.

The third example deals with the valuation of carbon dioxide. To mitigate the consequences of fossil fuel use, one can capture and store itin a geological depository. This is however a complicated and expensive process. Basic research is carried out in CEA on an alternative approach, developing a promising new method of catalytic conversion of $\mathrm{CO}_{2}$ into formamides. This method gets many advantages: the catalyst is cheap, the work is done at low pressure with no solvent nor additive. It would limit the use of oil in the synthesis of compounds such as glues, fertilizers or acrylic fibers. 


\section{As regards technological research, what could be done to meet the 2050 objectives?}

Transport and housing are intensive energy consumers and use mainly of fossil fuels. To decrease this dependence on fossil fuels, France, like some other countries has decided to build its mix on nuclear and renewable, with the use of fossil fuels as reduced as possible as soon as possible. Renewable energy could complete the base load ensured by the nuclear. The storage of electricity will allow adjusting production and consumption.

According to this statement, I think that technological research must be carried out in three main directions: optimized production of non GHG emission energy based on nuclear and renewable energies, energy storage and grid technologies, and reduction of energy consumption.

\section{What about energy production?}

The potential renewable energies in EU are numerous: solar energy, wind energy, bio-fuels and hydraulic energy including wave and tidal power without forgetting the potential of geothermal energy resources.

The main issues to be addressed by research in this field are to reduce the cost of the production of the needed transfer systems and to improve their performance, mainly in the field of systems life-time and maintenance when we speak about renewable sources.

- In solar energy, we must limit the quantity of materials in the detectors and look for abundant and non-toxic materials. The main goal is to produce in Europe and an economic objective, for example to reach $0.5 € / \mathrm{W}$ for the module and an efficiency of $25 \%$ by 2030 .

- In wind and marine energy, we target to optimize floating wind turbines and identify efficient technologies for marine stream and wave energy. For offshore systems, we have to connect turbines to high voltage grids and to develop maintenance structures.

- In bio-fuels we have to evaluate the resource, the techno-economic process and the impact. We must optimize pre-industrial processes in $2^{\text {nd }}$ generation systems which a way to store renewable energies as biodiesel and jet fuel coming from the coupling of biomass gazeification and hydrogen production from water splitting with electricity coming renewable or nuclear sources. And finally we have to develop growing, harvesting and extraction processes for $3^{\text {rd }}$ generation bio-fuels and conversion of microalgae biomass in bio-energy. 
Concerning nuclear energy, the Sustainable Nuclear Energy Technology Platform (SNETP) promotes research, development and demonstration of the nuclear fission technologies necessary to achieve the SET-Plan goals:

- For 2020, the aim is to maintain safety and competitiveness in fission technology and to provide long-term waste management solutions.

- For 2050, we act now to complete the demonstration of a new generation (Gen IV) of fission reactors with increased sustainability and to enlarge nuclear fission applications beyond electricity production.

The first challenge resulting is to support the current fleet of $2^{\text {nd }}$ and $3^{\text {rd }}$ generation reactors. We have to promote the extension of their life operation with the highest level of safety, mainly after Fukushima. Think about that in France, the electricity cost from 30 years old nuclear power plants is now $90 \%$ associated with investment cost and only $10 \%$ on operation cost including waste management and decommissioning. The economy is even better if you expend the NPP lifetime to 40 or 50 years. Lessons from the Fukushima accident show that efforts are needed to understand better the mechanisms in a severe accident and the way to mitigate the consequences; for instance, the hydrogen risk, the estimation of the released radioactivity and the seismic assessment. For the nuclear fuel cycle, research will aim to improve the processing and the recycling of used fuels, their storage and the final geological disposal.

The second challenge is to develop the $4^{\text {th }}$ generation of nuclear reactors. This research will meet the objective of a sustainable nuclear energy based on the highest safety standards. Such reactors will optimize the use of uranium and plutonium produced in the LWRs. Indeed, we use presently only $0,6 \%$ of the energy content of the uranium ore we extract from the ground while with this next generation of fast neutrons reactors we expect to use up to 100 times more. Furthermore, through the transmutation of minor actinides, the toxicity of ultimate wastes could be reduced as well.

The European Sustainable Nuclear Industrial Initiative (ESNII) created by the SNETP in support to the SET Plan will address the need for demonstration of Gen-IV Fast Neutron Reactor technologies. It is proposed in the roadmap to study in parallel several technologies: the sodium-cooled fast neutron reactor technology is the reference solution, with the construction of a prototype called ASTRID around 2020 in France. Alternative technologies - either lead-cooled or gas-cooled fast reactor - will be hosted in other European countries with the support of France. 
The third challenge is to promote a sustainable nuclear energy, carrying out research on the geological disposal of ultimate nuclear wastes as well as decommissioning and dismantling of nuclear facilities.

The main technological barriers to overcome concern the reactor physics the improvement of the fuel cycle, the waste management, the nuclear instrumentation, the command control and the development of advanced materials with numerical simulation as a key driver. Such research will require the use of large research infrastructures like material testing reactors, critical facilities, hot laboratories, fuel fabrication facilities and high performance computing centres.

\section{What about electricity storage and grid technology?}

A specific attention must be given to the electricity storage and grid developments to mitigate the consequences of the intermittence of renewable.

Energy storage is a key priority for the integration of renewable in the mix, especially for the wind of which average available power for European countries is in the range of $20 \%$ of the installed one with large unpredictable variations along the day, the month and the year. For example, for France in 2010, that wind generation was almost nil for 5\% of the time and does exceed $70 \%$ of installed capacity for only a few percent of the time. With an increasing share of the renewable, this explains the difficulty of managing the grid and the need for back-up capacities. Current systems to massively store electricity rely presently on pumping water between tanks, which is limited. It is of first importance to develop storage capacities based on new concepts and on improved grid management : CEA is strongly committed in this way. Local production, local storage and local consumption concept must be fostered, avoiding too large flows on the grids and making it easier.

I would like to underline that this storage issue has been clearly identified at the European level. It is the subject of a joint research program launched in November 2011 by the EERA executive committee.

For the future, in addition to pumping systems, the identified concepts are Compressed Air Energy Storage, innovative batteries, flywheels, hydrogen and thermal storage. For the hydrogen solution, research will concern the development of innovative electrolyser and fuel cells.

For mobile applications (transport), solutions rely on the use of reliable and safe batteries or aboard hydrogen storage. For the Li-Ion batteries the target is, for 20000 cycles, to reach a 
production cost of 200 to $300 € / \mathrm{kW}$, in an economic competition where prices quickly decrease. The objectives for the hydrogen consist in improving the capacity of the tanks while decreasing their costs.

For micro storage (electronic devices), two kinds of system are considered, the batteries and the hydrogen associated with fuel cells. This market is quickly increasing. Research aims to improve the capacities of these systems while reducing their cost.

\section{The third direction to explore for technological research: Energy efficiency}

"Energy efficiency" includes two aspects and could significantly reduce energy expenses: the energy sobriety of individuals, the social organisation, the energy performance of technologies and systems.

High energy prices reflecting the real costs of the different energies and new standards may contribute to promote energy efficiency but research remains the most efficient way for the long term to overcome technological barriers.

Research on the energy efficiency concerns mainly the active efficiency for energy flow optimization. Storage technologies are involved in this approach. Other aspects concern heat recovering and recycling and smart grid development and deployment. The use of low consumption ICT components at the various levels of the grid enables adapting the production to the consumption and promoting the consumption when energy is cheap.

\section{Conclusion}

As a conclusion, I would like to highlight some issues.

A sustainable energy supply is of major importance for the future of Europe for obvious environmental, social and economical reasons. Our joint efforts have to be as high as the stakes. But we should be cautious when joining our efforts not to give into the temptation to focus in a too restricted number of technologies. There is no single way to tackle the energy issue within Europe. For that reason I think it would be a mistake to try to find a unique solution. Our diversity is a chance, a chance to share risks, a chance to share benefits. Intensive research is essential to reach our goals. Our strategy has to be based on three principles: neutrality towards all the energies which comply with our common goals, openness to partnership as far as technological choices are concerned, willingness to take advantages of complementarities.

It will be the best chance to reach the objective of EU for 2050 ! 